\title{
Erratum
}

\section{Prediction Model of Conversion to Dementia Risk in Subjects with Amnestic Mild Cognitive Impairment: A Longitudinal, Multi-Center Clinic-Based Study}

Hyemin Jang, Byoung Seok Ye*, Sookyoung Woo, Sun Woo Kim, Juhee Chin, Seong Hye Choi, Jee Hyang Jeong, Soo Jin Yoon, Bora Yoon, Kyung Won Park, Yun Jeong Hong, Hee Jin Kim, Samuel N. Lockhart, Duk L. Na and Sang Won Seo

[Journal of Alzheimer's Disease 60(4), 2017, 1579-1587, DOI: 10.3233/JAD-170507]

https://content.iospress.com/articles/journal-of-alzheimers-disease/jad170507

On page 1579, the affiliation for the author Samuel N. Lockhart was incorrectly given. The correct affiliation for Samuel N. Lockhart is:

${ }^{\mathrm{i}}$ Department of Internal Medicine, Wake Forest School of Medicine, Winston-Salem, NC 